\title{
Low Subfrontal Dural Opening for Anterior Clinoid Meningioma
}

\author{
Michael R. Chicoine ${ }^{1}$ \\ ${ }^{1}$ Department of Neurosurgery, Washington University, St. Louis, \\ Missouri, United States
}

J Neurol Surg B 2018;79(suppl S3):S273-S275.

\author{
Address for correspondence Michael R. Chicoine, MD, Department of \\ Neurosurgery, Washington University, 660 S. Euclid campus Box \\ 8057, St. Louis, MO 63110, United States
} (e-mail: chicoinem@wudosis.wustl.edu).

\begin{abstract}
Keywords

- low subfrontal approach

- meningioma

- anterior clinoid process

- extradural dissection

Introduction A variety of dural openings are described for frontal-temporal and cranioorbital craniotomies. As with any surgical technique, the goal is to optimally and safely address the pathology, minimize normal anatomy disruption, and optimize postoperative recovery. This study reports a modified dural opening for frontal-temporal approaches which minimizes brain exposure while facilitating visualization for neoplastic and vascular lesions of the anterior clinoid, supra- and parasellar and adjacent regions.

Methods A sample case is presented for which a low subfrontal dural exposure was utilized for tumor resection. The clinical presentation, surgical procedure, and outcome are summarized including a video detailing the surgical technique.

Results A 63-year-old female with gradual left eye vision loss and tumor enlargement on serial imaging. The small dural-based tumor arose from the left anterior clinoid and optic canal region compressing the optic nerve. The video shows a left frontaltemporal craniotomy used to perform extradural anterior clinoidectomy and optic canal decompression. The novel low subfrontal dural opening without fixed brain retraction allowed dural opening around the optic canal and clinoidectomy region to resect tumor and complete optic nerve decompression. Postoperatively, the patient did well with significant recovery of vision and follow-up at 2.5 years shows no evidence of tumor recurrence.

Conclusion The novel low subfrontal dural opening provides access to lesions of the supra-and para-sellar and adjacent regions, and preservation of much of the dura avoids some of the risks of intradural dissection including Sylvian fissure dissection and brain retraction

The link to the video can be found at: https://youtu.be/Jc7wvR4PTFk.
\end{abstract}

Conflict of Interest

None.

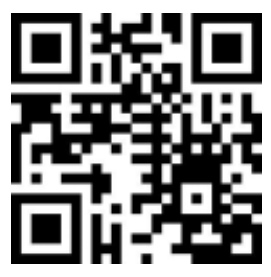

received

October 7, 2017

accepted

December 12, 2017

published online

February 8, 2018

www.thieme.com/skullbasevideos

www.thieme.com/jnlsbvideos

DOI https://doi.org/

10.1055/s-0038-1624586. ISSN 2193-6331.
๑) 2018 Georg Thieme Verlag KG
Stuttgart · New York

License terms

(c) (1) $\ominus$ (\$) 


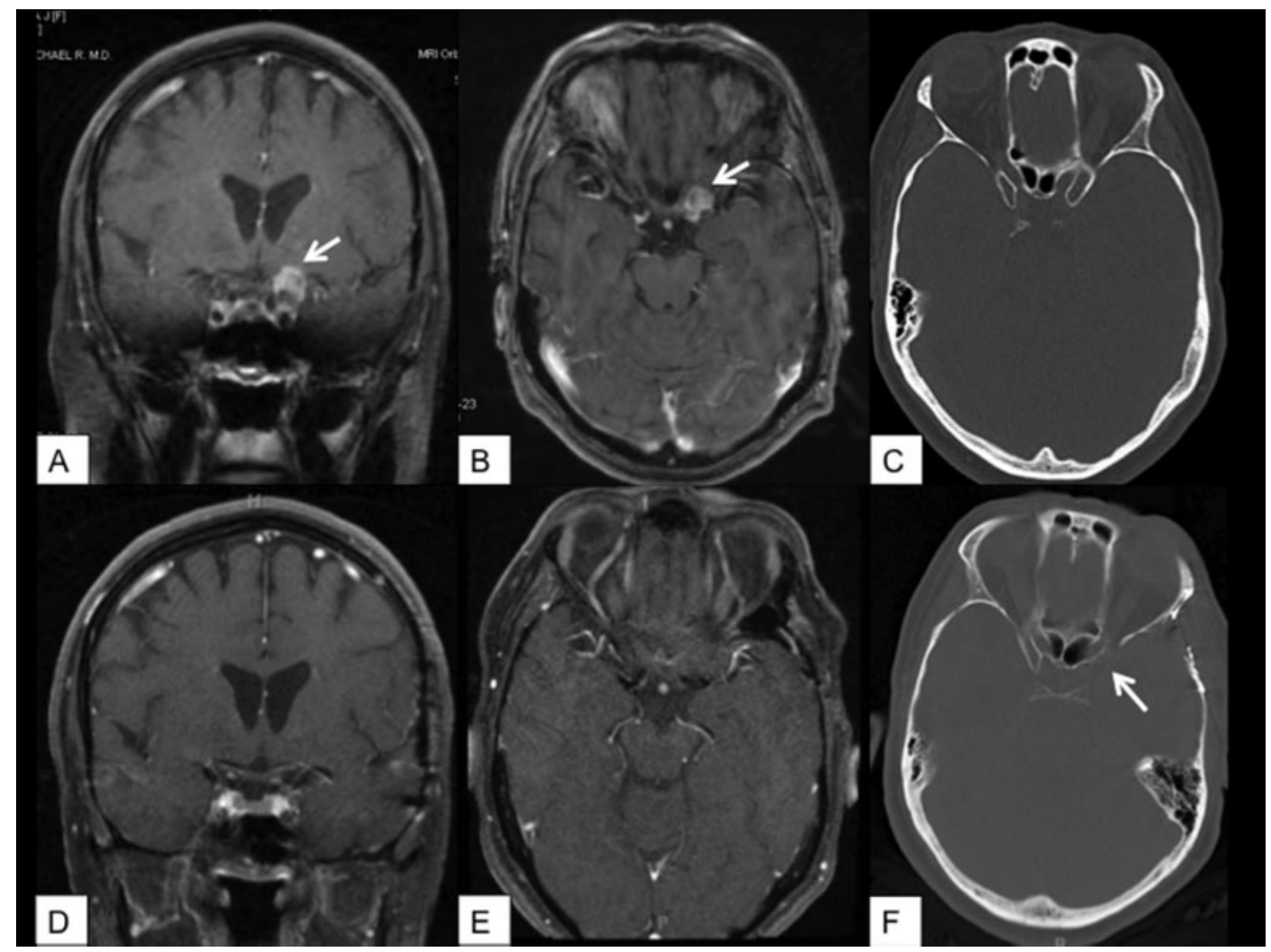

Fig. 1 (A and B) Preoperative magnetic resonance imaging (MRI) with arrows demonstrating the dural-based anterior clinoid and optic canal region lesion. (C) Preoperative computed tomography (CT). (D and E) Postoperative MRI demonstrating lesion resection. (F) Postoperative CT with arrow identifying the anterior clinoidectomy. 

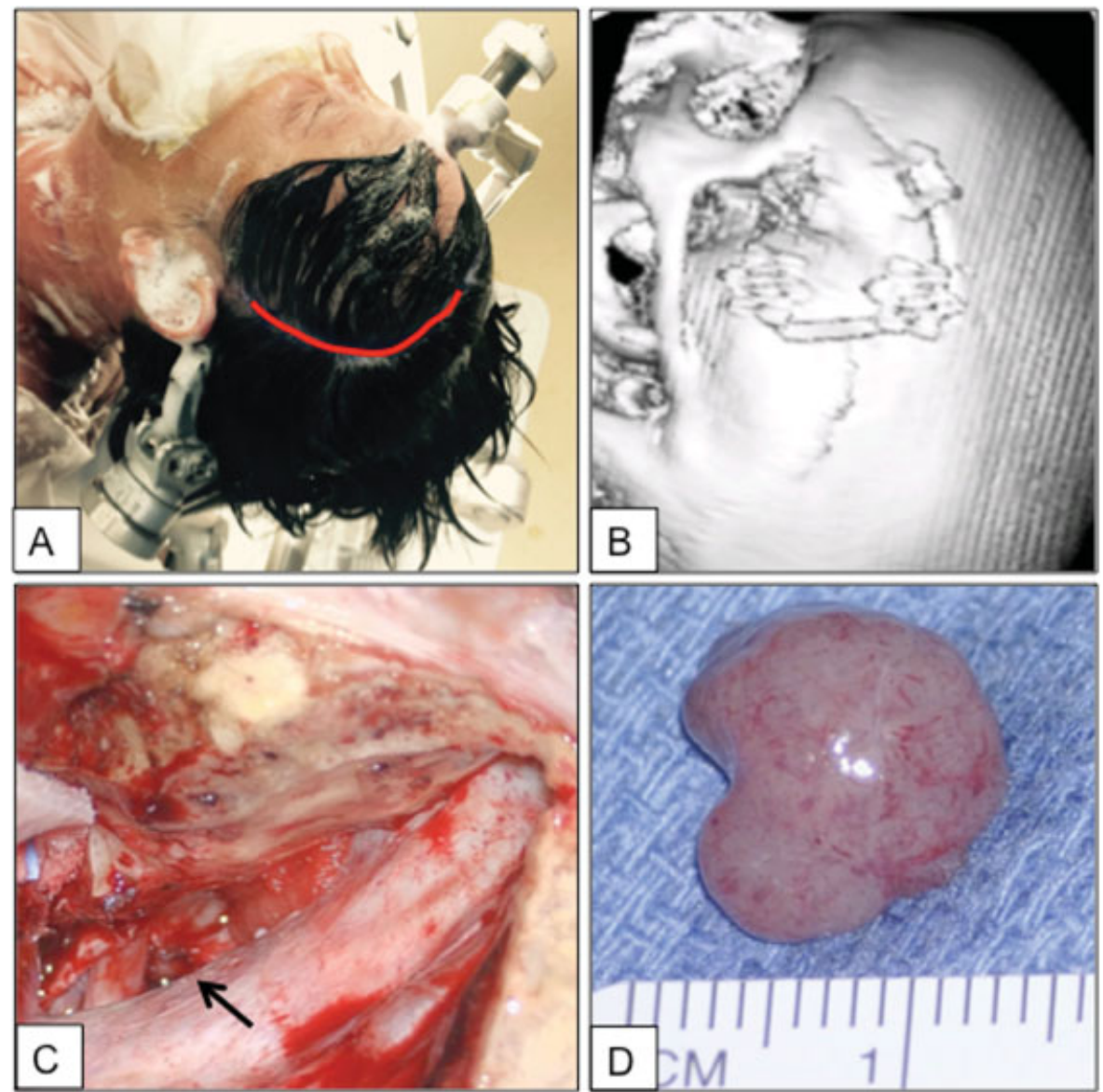

Fig. 2 (A) The patient is positioned supine in headholder with head rotated to right and extended to facilitate frontal lobe shift away from the floor of the anterior fossa. Hair-sparing left frontal-temporal incision is approximated by the red line. (B) Postoperative three-dimensional computed tomography reconstruction showing the extent of the craniotomy. (C) Intraoperative microsurgical image showing the low subfrontal dural opening (arrow) permitting visualization of the decompressed left optic nerve after anterior clinoidectomy, optic canal osteotomy, and resection of the left anterior clinoid meningioma. Much of the frontal-temporal dura remains intact and no self-retaining retractors are used thereby avoiding some of the risks of Sylvian fissure dissection and other aspects of wider intradural exposure. (D) Small meningioma tumor specimen largely removed en bloc. 